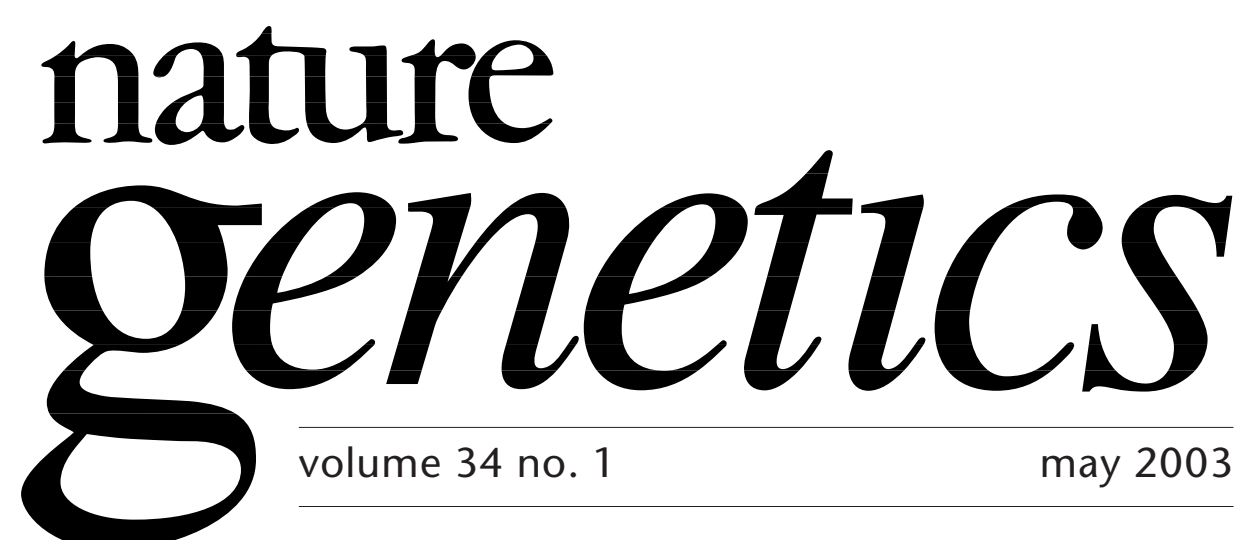

\title{
From the ground up
}

The room within is the great fact about the building.

-Frank Lloyd Wright

Science, like time, waits for no one. The rapid pace of discovery means that resources are exploited as soon as they are generated, well before completion, and often in unexpected ways. This is certainly true for genomic sequence data, which are made available to the wider community almost immediately. There are moments, however, when the overall landscape is assessed and new goals are set, even as the work continues. The publication of $A$ Vision for the Future of Genomics Research in the 24 April issue of Nature marks one such occasion.

In 1988, the National Research Council published its landmark report, Mapping and Sequencing the Human Genome, which made the first formal case for what came to be known as the Human Genome Project (HGP). Subsequent reports in 1990, 1993 and 1998 by the US Department of Energy and National Human Genome Research Institute (NHGRI) provided updates on progress and suggested mid-course corrections. With the sequence in hand, thanks to the justly celebrated efforts of an international public consortium and an enterprising private company, an agenda for the next decade and beyond is now in the offing.

Written by Francis Collins, Eric Green, Alan Guttmacher and Mark Guyer on behalf of the NHGRI, and informed by nearly two years of consultations with the National Advisory Council for Human Genome Research and hundreds of scientists and interested members of the public, $A$ Vision outlines a way forward for genomics. Although there is far too much to comment on in this space, a few points seem especially worth highlighting.

The authors compare the themes that they're proposing to three floors of a building, resting on the foundation of the HGP, and the whole structure being held together by six 'crosscutting elements'. The overarching themes are 'genomics to biology,' 'genomics to health' and 'genomics to society', whereas the crosscutting elements constitute the necessary advances in resources, technology development, computational biology, training, ethical, legal and social questions, and education.

The application of genomics to biology is, of course, well underway, and is spelled out here as a series of 'grand challenges', the first of which is the need to generate a complete description of the structural and functional elements of the human genome. One systematic effort to accomplish this is the proposed Encyclopedia of DNA Elements (ENCODE) program, which aims to use all available approaches to identifying genes, splice variants, promoters, enhancers, origins of replication and determinants of chromosome structure. 
Needless to say, this is an immense task. This is particularly true when it comes to the characterization of regulatory elements, which, if the results from model organisms are any guide, can influence gene expression in complex ways and from unpredictable locations in the genome. A pilot project, soon to be launched, will focus on $30 \mathrm{Mb}$ of the human genome (about 1\%). These sequences have been chosen partly at random, and partly with intent, based on the presence of wellstudied genes and the availability of comparative sequence data. The pace of progress in this effort should be an early signpost as to the likelihood that a 'deep' functional annotation of the genome can be generated in the foreseeable future.

A robust genomic medicine is obviously a long-term goal, which will require a greater interest in drug design and the pathophysiology of disease on the part of basic scientists, and a greater appreciation of genomics on the part of clinicians. One noteworthy angle to be found in the NHGRI blueprint is an emphasis on the identification of gene variants that contribute to good health. This view of the field tends to get less attention than the search for alleles that are associated with disease, but the authors rightly propose a concerted effort to identify protective alleles in 'healthy cohorts'. Specifically, they suggest looking at individuals who are at high risk for developing a certain disease who nonetheless remain healthy (individuals with mutations in HNPCC who do not develop colon cancer, for example).

The application of genomics to health care will also no doubt be hastened by the emerging field of phenomics, as discussed on page 15 of this issue by Nelson Freimer and Chiara Sabatti. A serious attempt to develop high-throughput methods for collecting standardized phenotypic information will be needed to ensure that genotypic information will be properly interpreted and applied. Collins et al. and Freimer and Sabatti agree on the importance of studying longitudinal population cohorts in this regard, such as the Avon Longitudinal Study of Parents and Children, which has been gathering data on genetic, phenotypic and environmental variables from several thousand families in southwestern England. Among the questions being addressed with some degree of success is the interaction between genetic variants and maternal nutrition in influencing fetal growth, which is a known risk factor for type 2 diabetes, hypertension and cardiovascular disease.

There are many other avenues of research described in A Vision, along with a few provocative 'blue sky' goals (the $\$ 1,000$ whole-genome sequence, for example) and a continuing emphasis on the ethical, legal and social ramifications of genomics. Overall, the 'building' that the authors have in mind is, of course, a virtual one; nonetheless, the sketch on the first page of their proposal bears a striking resemblance to one of the most admired architectural achievements of the $20^{\text {th }}$ century-Frank Lloyd Wright's Fallingwater. Perched on a waterfall that feeds into a mountain stream in a wooded region of western Pennsylvania, Fallingwater is a private residence that was built for the family of Edgar J. Kaufmann in the 1930s. In 1986, architecture critic Paul Goldberger wrote the following:

This is a house that summed up the $20^{\text {th }}$ century and then thrust it forward still further. Within this remarkable building Frank Lloyd Wright recapitulated themes that had preoccupied him since his career began a half-century earlier, but he did not reproduce them literally. Instead, he cast his net wider... and pulled it all together into a brilliantly resolved totality.

Praise like that is reserved for buildings, not blueprints. But if the advances foreseen in this ambitious document come to pass, then all involved will one day deserve it. 